\title{
Autonomic Status Epilepticus in a Patient with Parasellar Meningioma: A Case Report
}

\author{
Giacomo Evangelista $^{a}$ Fedele Dono $^{a}$ Stefano Consolia Dario Calisi ${ }^{a}$ \\ Marco Onofrj ${ }^{a}$ Francesca Anzellotti $^{b}$ Valerio Frazzini ${ }^{c, d}$ Laura Bonannia \\ aDepartment of Neuroscience, Imaging and Clinical Science, "G. D'Annunzio" University of \\ Chieti-Pescara, Chieti, Italy; 'Department of Neurology, Epilepsy Center, "SS Annunziata" \\ Hospital, Chieti, Italy; 'AP-HP, Epilepsy Unit, Pitié-Salpêtrière Hospital, Sorbonne University, \\ Paris, France; ${ }^{\mathrm{d} B r a i n}$ and Spine Institute (INSERM UMRS1127, CNRS UMR7225, Sorbonne \\ Université), Pitié-Salpêtrière Hospital, Paris, France
}

\section{Keywords}

Nonconvulsive status epilepticus · Aura continua $\cdot$ Brain tumor $\cdot$ Case report

\section{Abstract}

Autonomic status epilepticus (Aut SE) is a condition characterized by ongoing focal autonomic seizure lasting for $>30 \mathrm{~min}$. Aut SE can show a variety of clinical manifestations including vomiting, nausea, changes in heart rate, piloerection, pupillary abnormalities, and visual abnormalities. Although Aut SE is a common finding in childhood in the context of Panayiotopoulos syndrome, few reports have described this condition during adulthood. In the present report, we describe a case of Aut SE in an adult patient with parasellar meningioma and bilateral frontotemporal epileptiform activity on EEG record.

(C) 2021 The Author(s).

Published by S. Karger AG, Basel

\section{Introduction}

Autonomic status epilepticus (Aut SE) is a subtype of nonconvulsive status epilepticus (NCSE) characterized by ongoing focal autonomic seizures [1]. Various clinical manifestations can be associated with Aut SE, including vomiting, nausea, piloerection, heart rate changes, pupillary alterations, and "abdominal feeling," often described as a rising sensation [1]. Aut SE usually occurs during childhood in the context of Panayiotopoulos syndrome (PS), 
but rare cases during adulthood have been reported [1]. Even though in PS ictal EEG shows clear characteristics (i.e., high-amplitude sharp and slow-wave abnormalities intermixed with small spiky activity mainly localized in occipital area) [1], EEG features in adults are more nonspecific in terms of localization and morphology of epileptiform abnormalities, which are more likely related to the patient's epilepsy type rather than being a characteristic of the autonomic nature of the seizures [1]. In the present report, we describe a case of Aut $\mathrm{SE}$ in an adult patient suffering from brain tumor, whose EEG recording showed an unusual frontotemporal bilateral epileptiform activity.

\section{Case Report}

A 36-year-old man was admitted to the emergency room complaining of the abrupt onset of epigastric pain, intense abdominal discomfort, and cognitive-motor slowing. His medical history showed multiple neoplasia (including a recent diagnosis of colorectal cancer and right clear cell renal cell carcinoma at the age of 35 and right suprarenal neuroblastoma treated during childhood), type 1 diabetes mellitus, dysthyroidism, hypogonadism, and renal failure (stage IV kidney disease). No prior history of epilepsy was reported. Familial medical history was unremarkable. Genetic tests for multiple endocrine neoplasia syndrome as well as familial adenomatous polyposis were negative. The last oncological follow-up showed no metastatic disease.

At the admission, the patient referred abnormal unremitting abdominal "feeling" described as a rising sensation, and the neurological evaluation showed speech difficulties and ideomotor slowing without impaired awareness which had started $6 \mathrm{~h}$ before. Similar episodes with variable frequency and short-lasting duration were reported during the past 3 days. The patient underwent a brain CT which showed an extra-axial neoplasia of unknown etiology. Concomitantly with the aforementioned clinical symptoms, a standard 21-electrodes electroencephalogram (EEG) showed continuous, rhythmic, bilateral spike-and-slow-wave complexes of frontal origin at $2-2.5 \mathrm{~Hz}$ and $100-150 \mathrm{mV}$ of amplitude (Fig. 1a). A bipolar electrocardiogram (EKG) recording from lead I of a 12-lead EKG was performed using the EKG channel of the EBN-Neuro EEGNet System (EBN-Neuro, Florence, Italy) which showed moderate bradycardia (heart rate, $\mathrm{HR}=57$ beats per minute). Heart rate variability (HRV) analysis was carried out using a dedicated software (Kubios, HRV software; University of Eastern Finland, Kuopio, Finland) which showed increased high frequency values ( $\left.\mathrm{HF}=1,090 \mathrm{~ms}^{2}\right)$ in frequencydomain analysis, increased root mean square of successive differences (RMSSD $=56.8 \mathrm{~ms}$ ) in time-domain analysis, and increased standard deviation 1 (SD1: $40.2 \mathrm{~ms}$ ) and 2 (SD2: $99.8 \mathrm{~ms}$ ) in the Poincare plot of the nonlinear analysis (Fig. 2a). These results showed an increased cardiovagal output during the SE.

According to Salzburg criteria, a diagnosis of NCSE was made. Thus, the patient was treated with an intravenous bolus of levetiracetam (LEV) $500 \mathrm{mg}$ followed by a prompt clinical improvement in the next $30 \mathrm{~min}$. Considering the good clinical response to the first LEV bolus, taking into account patient's renal function (creatinine clearance: $<30 \mathrm{~mL} / \mathrm{min}$ ), we did not administrate further LEV doses. A regimen of oral daily administration of LEV $1000 \mathrm{mg} /$ day was subsequently implemented, with no side effects. On the basis of the electroclinical response, a serial 60-min EEG monitoring was performed every $24 \mathrm{~h}$. At the first control, the EEG showed interictal abnormalities consisting of high-amplitude 2- to 3-Hz delta activity in the bilateral frontotemporal derivations (Fig. 1b). MRI of the brain showed a left, contrast-enhanced, parasellar lesion compressing the left temporal lobe (Fig. 3a, b). According to the neuroradiological features, a diagnosis of parasellar meningioma was made. Two weeks later, EEG showed slow abnormalities in the bilateral frontal and left temporal derivations (Fig. 1c).

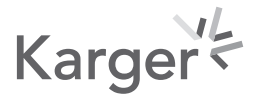




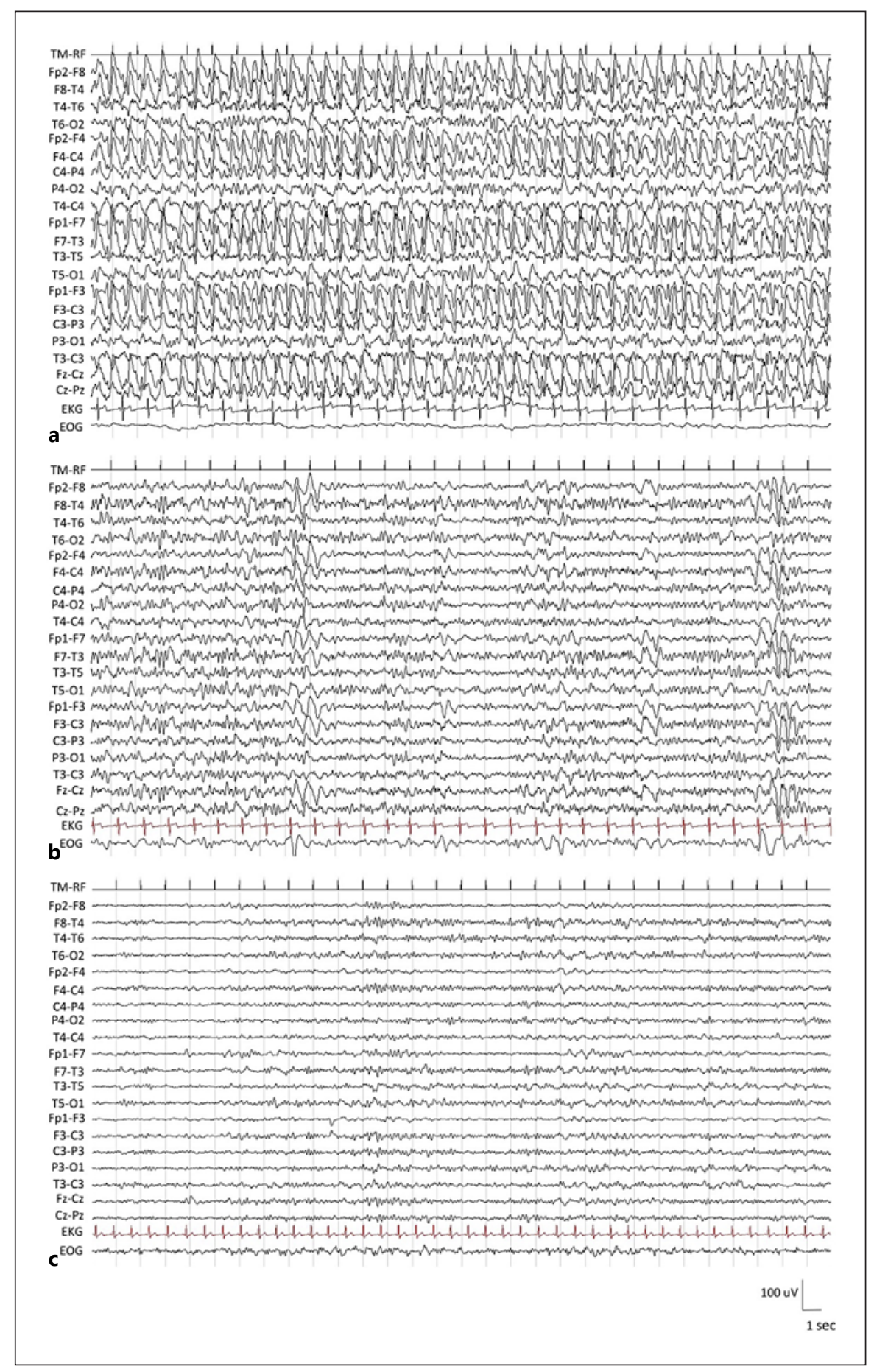

Fig. 1. EEG. a EEG performed during the ictal phase: continuous bilateral spike-and-slow-wave complexes occurring synchronously in the frontal derivations at 2-2.5 Hz and 100-150 mV of amplitude. b EEG performed after $24 \mathrm{~h}$ showed high-amplitude 2 - to 3 -Hz delta activity in the bilateral frontotemporal derivations. c EEG performed after 2 weeks showed normal background alpha activity with sporadic $4-5 \mathrm{~Hz}$ slowing in the bilateral frontotemporal derivations. 


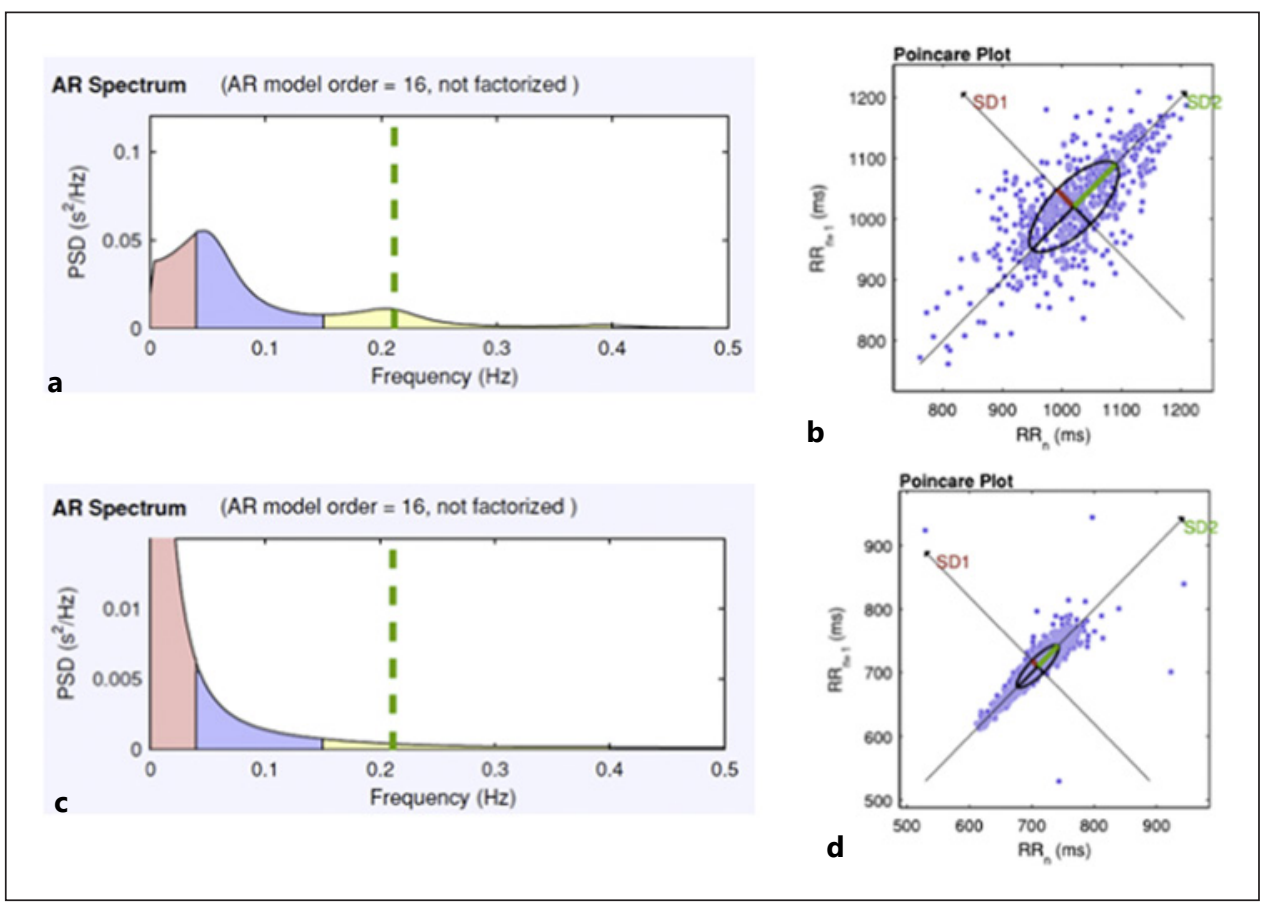

Fig. 2. Heart rate variability frequency-domain analysis and nonlinear result. Frequency-domain analysis was performed using autoregressive methods. In a typical short-time $(\cong 5 \mathrm{~min})$ spectral analysis, the spectral power is divided into 3 frequency bands: HF (0.15-0.40 Hz) (yellow), LF (0.04-0.15 Hz) (blue), and VLF (0-0.04 Hz) (red). The Poincare plot of RR intervals reflects the nonlinear complexity of the HR signal. The Poincare plot is a graph of each successive RR (or NN) interval versus the next. a Frequency-domain analysis and the Poincare plot during the acute phase of the Aut SE (b). c Frequency-domain analysis and the Poincare plot after the resolution of the Aut SE (d). HF, high frequency; LF, low frequency; VLF, very low frequency.

A concomitant ECG showed normal HR (84 beats per minute), whereas HRV analysis, performed in time-domain (RMSSD) and frequency-domain (HF), as well as in nonlinear analysis (SD1 and SD2), showed reduction of values of RMSSD (18.8 ms), HF (80 ms $\left.{ }^{2}\right), \operatorname{SD} 1$ (13.3 ms), and SD2 (46.3 ms) of the previously recorded abnormal vagal tone (Fig. 2b).

\section{Discussion}

Aut SE is a subtype of NCSE typically described during childhood in the context of PS [1]. Some previous reports of Aut SE in adulthood have been labeled as cases of "aura continua," a condition described as a "curious seizure of long duration" with recurring episodes of a continuous epigastric sensation [2]. The temporal lobe plays a central role in determining the onset of autonomic symptoms during seizures. In fact, episodes of "aura continua" have been reported in the context of temporal lobe epilepsy.

In our report, we describe a case of Aut SE characterized by "aura continua" and an increased vagal tone with bradycardia (as shown by HRV analysis and ECG recording), associated with rhythmic bifrontal predominant spike-and-wave activity at the EEG. The HRV analysis is a valuable diagnostic tool to assess cardiac autonomic control [3]. The HRV provides information on the variations of instantaneous R-R intervals of the ECG. Time-domain (i.e., RMSSD) and frequency-domain (i.e., HF) parameters can be calculated on the basis of a short ECG recording (5-min length) and give important information with regard to vagal output [4]. 

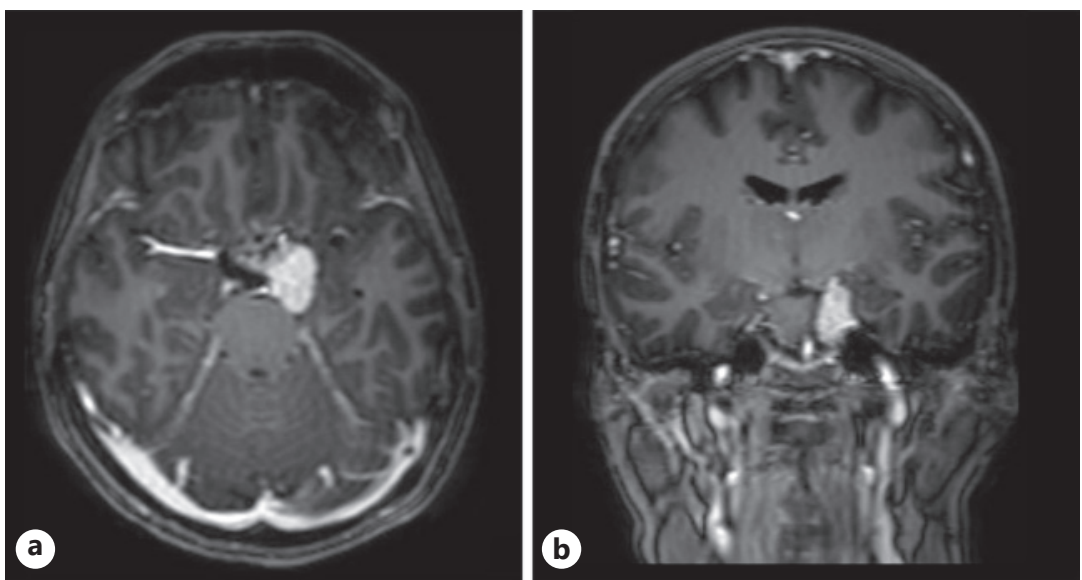

Fig. 3. MRI. a Axial and $\mathbf{b}$ coronal T1-weighted MRI scans of the brain show a left parasellar meningioma that compressed the left mesial temporal lobe and the left cisterna pontocerebellaris.

The analysis of HRV has been extensively employed to ascertain changes in the sympathovagal balance of the physiological response exhibited by either healthy subjects or patients affected by cardiac or neurological diseases such as epilepsy [5]. In our report, we stressed the importance of HRV evaluation which can support diagnosis of Aut SE and distinguish it from other forms of NCSE. However, it has to be acknowledged that seizures whether clinical or subclinical can be associated with HRV alteration without any specificity to seizure type.

This clinical picture can be explained by the specific brain MRI findings. In particular, the presence of brain tumor which compresses the left mesial temporal lobe and the subsequent ongoing epileptic activity emerging from this site can define the peculiar epigastric sensation and the increased cardiovagal tone [6]. Furthermore, bilateral frontotemporal epileptic discharges can result from the rapid spread and organization of epileptic activity in the frontal lobes [7].

\section{Conclusion}

Diagnosis of Aut SE in adulthood is challenging since autonomic symptoms can be easily misinterpreted as nonneurological manifestations especially in patients without a history of epilepsy. However, the association of autonomic symptoms with brain lesions should rise the diagnostic suspect. EEG findings in the context of Aut SE can be varied and may include frontotemporal bilateral epileptiform discharges. The prompt recognition of Aut SE and initiation of antiseizure treatment can prevent seizure refractoriness and brain damage.

\section{Statement of Ethics}

Written informed consent was obtained from the patient for publication of this case report and any accompanying images. The study is exempt from ethics committee approval because it is not necessary for the publication of the case report.

\section{Conflict of Interest Statement}

Authors declare no conflicts of interest.

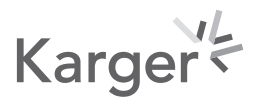




\section{Funding Sources}

There are no funding sources to report.

\section{Author Contributions}

G.E. and F.D. contributed to the conception and design of the study. G.E., F.D., and S.C. wrote the manuscript. D.C., M.O., F.A., V.F., and L.B. contributed to manuscript revisions, read, and approved the submitted version.

\section{Data Availability Statement}

The data are available from the corresponding author upon reasonable request.

\section{References}

1 Ferrie CD, Caraballo R, Covanis A, Demirbilek V, Dervent A, Fejerman N, et al. Autonomic status epilepticus in Panayiotopoulos syndrome and other childhood and adult epilepsies: a consensus view. Epilepsia. 2007 Jun; 48(6):1165-72.

2 Seshia SS, McLachlan RS. Aura continua. Epilepsia. 2005 Mar;46(3):454-5.

3 Malik M, Bigger JT, Camm AJ, Kleiger RE, Malliani A, Moss AJ, et al. Heart rate variability standards of measurement, physiological interpretation, and clinical use. Eur Heart J. 1996;17:354-81.

4 Laborde S, Mosley E, Thayer JF. Heart rate variability and cardiac vagal tone in psychophysiological research: recommendations for experiment planning, data analysis, and data reporting. Front Psychol. 2017;8:213.

5 Romigi A, Albanese M, Placidi F, Izzi F, Mercuri NB, Marchi A, et al. Heart rate variability in untreated newly diagnosed temporal lobe epilepsy: evidence for ictal sympathetic dysregulation. Epilepsia. 2016 Mar;57(3): 418-26.

6 Dono F, Evangelista G, Frazzini V, Vollono C, Carrarini C, Russo M, et al. Interictal heart rate variability analysis reveals lateralization of cardiac autonomic control in temporal lobe epilepsy. Front Neurol. 2020 Aug 14;11: 842.

7 Lin JJ, Riley JD, Juranek J, Cramer SC. Vulnerability of the frontal-temporal connections in temporal lobe epilepsy. Epilepsy Res. 2008 Dec;82(2-3):162-70. 